\title{
Asset Trading Volume in Infinite-Horizon Economies with Dynamically Complete Markets and Heterogeneous Agents: Comment
}

\author{
Peter Bossaerts* \\ California Institute of Technology \\ Centre for Economic Policy Research \\ William R. Zame ${ }^{\dagger}$ \\ UCLA \\ California Institute of Technology
}

March 21, 2005

\footnotetext{
${ }^{*}$ Financial support from the R. G. Jenkins Family Fund and the National Science Foundation is gratefully acknowledged. Opinions, findings, conclusions and recommendations expressed in this material are those of the authors and do not necessarily reflect the views of any funding agency.

$\dagger$ Financial support from the John Simon Guggenheim Memorial Foundation, the National Science Foundation, the Social and Information Sciences Laboratory at Caltech, and the UCLA Academic Senate Committee on Research is gratefully acknowledged. Opinions, findings, conclusions and recommendations expressed in this material are those of the authors and do not necessarily reflect the views of any funding agency.
} 


\title{
Asset Trading Volume in Infinite-Horizon Economies with Dynamically Complete Markets and Heterogeneous Agents: Comment
}

\begin{abstract}
In a recent paper, Judd, Kubler \& Schmedders (2003) study asset trading in a version of the standard Lucas infinite horizon economy with heterogeneous agents. They report the surprising finding that (for generic economies in their class), in equilibrium, there is no trade in (long-lived) assets after the initial date. This note points out that the conclusions of Judd, Kubler \& Schmedders (2003) are artifacts of the assumption that asset dividends and individual endowments follow the same stationary finite state Markov process. Without this assumption - and even if asset dividends and aggregate endowments follow the same stationary process - there will necessarily be trade at many histories.
\end{abstract}

JEL Classification Numbers D51, G12

Keywords Lucas asset pricing model, asset volume 
In a recent paper, Judd, Kubler \& Schmedders (2003) (hereafter JKS) study asset trading in a version of the standard Lucas (1978) infinite horizon economy with heterogeneous agents. They report the surprising finding that (for generic economies in their class), in equilibrium, there is no trade in (long-lived) assets after the initial date. They conclude that their result "indicates that other factors considered in the literature, such as life-cycle factors, asymmetric information, heterogeneous beliefs, and incompleteness of the asset market, play a significant role in generating trade volume."

The formal JKS no-trade result is correct, but we find the interpretation misleading. The standard Lucas (1978) model posits a finite number of assets whose dividends follow a stationary finite state Markov process and a representative agent whose endowment follows the same stationary process. JKS introduce heterogeneous agents but require that individual endowments also follow the same stationary process. It seems to us that the more natural heterogeneous agent version of the model should require only that aggregate endowments be stationary but allow for individual endowments that are not stationary. The latter assumptions yield the same conclusions about equilibrium prices and consumptions - stationarity in particular — but, as we show below, are consistent with portfolio trades at many dates, and even with portfolio processes that are not stationary, but rather depend on the entire history.

\section{The Asset Market Economy}

Our notation, model and assumptions are almost as in JKS; we recall them for the convenience of the reader. There is a single consumption good and an infinite horizon. Time is indexed by $t=0,1, \ldots$ Aggregate uncertainty follows a stationary finite state Markov process $\mathbb{M}$ with state space $\mathbb{S}=$ $\{1, \ldots, S\}$ and transition matrix $\Pi$; the initial state is 1 . We assume all entries of $\Pi$ (the transition probabilities) are strictly positive. A history of length $t$ is a sequence $\sigma_{t}=\left(y_{0}, \ldots, y_{t}\right)$ of realizations of $\mathbb{M}$; $y_{t}$ is the underlying state in the history $\sigma_{t}$. We write $\sigma_{t}^{-}=\left(y_{0}, \ldots, y_{t-1}\right)$ for the unique history that immediately precedes $\sigma_{t}$. Write $\Sigma_{t}$ for the set of histories of length $t$, $\Sigma=\bigcup_{t=0}^{\infty} \Sigma_{t}$ for the set of all histories, and 0 for the initial history.

$S$ long-lived assets are traded; dividends on these assets (which may be 
viewed as the outputs of unmodeled firms) are stationary (that is, follow the Markov process $\mathbb{M}$ ): the dividend $d^{j}\left(\sigma_{t}\right)$ on asset $j$ in history $\sigma_{t}$ depends only on the underlying state $y_{t}$ in the history $\sigma_{t}$. Hence, with no ambiguity we can write $d^{j}(y)$ for the dividend on asset $j$ in any history in which the underlying state is $y$. We assume the vectors $d^{1}(\cdot), \ldots, d^{S}(\cdot)$ of state-dependent dividends are linearly independent.

The economy is populated by $H$ investors (or types of investors). A consumption plan for an investor is a bounded function $x: \Sigma \rightarrow \mathbb{R}_{+}$. Investor $h$ maximizes expected discounted utility of consumption

$$
U_{h}(x)=E\left\{\sum_{t=0}^{\infty} u_{h}\left(x\left(\sigma_{t}\right), y_{t}\right)\right\}
$$

where utility $u_{h}$ for consumption may depend on the underlying state of the Markov process. We assume $u_{h}(\cdot, y): \mathbb{R}_{+} \rightarrow \mathbb{R}$ is smooth, strictly increasing, strictly concave, and satisfies the Inada condition $\frac{\partial u}{\partial x}(0, y)=\infty$. Investor $h$ 's endowment $e_{h}: \Sigma \rightarrow \mathbb{R}_{+}$is assumed to be bounded above and away from 0 . Note that agents may have different endowments and utility functions, but share the common discount factor $\beta$. In addition to claims to consumption, agents are endowed with portfolios of assets (shares in the firms $) ; \theta_{h}\left(0^{-}\right)$is agent $h$ 's endowment portfolio. Assets are in positive net supply, so $\sum_{h=1}^{H} \theta_{h}\left(0^{-}\right) \gg 0$.

If $\theta$ is a portfolio, we write $\operatorname{div}_{\sigma} \theta=\sum_{j=1}^{S} \theta^{j} d^{j}(\sigma)$ for the dividends of $\theta$ in the history $\sigma$. By assumption, dividends depend only on the underlying state, so we frequently write $\operatorname{div}_{y}$ instead of $\operatorname{div}_{\sigma}$ if $y$ is the underlying state in history $\sigma$.

We differ from JKS in assuming only that the aggregate endowment $\bar{e}=\sum_{h=1}^{H}\left[e_{h}+\operatorname{div} \theta_{h}\left(0^{-}\right)\right]$is stationary, so that $\bar{e}\left(\sigma_{t}\right)$ depends only on the underlying state $y_{t}$. For the moment we make no assumption about individual endowments.

Given asset prices $q: \Sigma \rightarrow \mathbb{R}^{S}$, agent $h$ 's budget set consists of consumption plans $x_{h}: \Sigma \rightarrow \mathbb{R}_{+}$, and portfolio plans $\theta_{h}: \Sigma \rightarrow \mathbb{R}^{S}$ such that:

$$
x_{h}\left(\sigma_{t}\right)=e_{h}\left(\sigma_{t}\right)+\operatorname{div}_{\sigma_{t}} \theta\left(\sigma_{t}^{-}\right)+q\left(\sigma_{t}\right) \cdot \theta_{h}\left(\sigma_{t}^{-}\right)-q\left(\sigma_{t}\right) \cdot \theta_{h}\left(\sigma_{t}\right)
$$


and $\sup _{\sigma_{t}} \mid\left(q\left(\sigma_{t}\right) \cdot \theta_{h}\left(\sigma_{t}\right) \mid<\infty\right.$. (The latter requirement eliminates doubling strategies.) A financial markets equilibrium consists of prices $\bar{q}: \Sigma \rightarrow \mathbb{R}^{S}$, consumption plans $\bar{x}_{h}: \Sigma \rightarrow \mathbb{R}_{+}$, and portfolio plans $\bar{\theta}_{h}: \Sigma \rightarrow \mathbb{R}^{S}$ such that each agent optimizes in his/her budget set and markets (for assets and hence consumption) clear.

\section{Portfolio Trades}

As JKS observe, at an efficient (Pareto optimal) allocation, all agents' marginal rates of substitutions are equal. Because the aggregate endowment is stationary if follows that, at any efficient allocation, individual consumptions must also be stationary. (See Lemma 1 in JKS or Section 20 in Duffie (1988).) It follows that, at any efficient financial markets equilibrium $\bar{q},\left(\bar{x}_{h}\right),\left(\bar{\theta}_{h}\right)$, asset prices $\bar{q}$ must also be stationary. If individual endowments are stationary — as assumed by JKS — it follows that agents achieve their equilibrium consumptions by trading assets only at the initial history: thereafter agents do not trade, but only consume their endowments and the dividends of the portfolio $\bar{\theta}_{h}(0)$.

What JKS do not observe is that the converse of their no-trade result is true as well: if individual endowments are not stationary then there must be trade after the initial date. To see this, observe that if agent $h$ does not trade after the initial date, then his/her consumption at history $\sigma_{t}$ will be the sum of consumption and dividends on the constant portfolio $\bar{\theta}_{h}(0)$ :

$$
\bar{x}_{h}\left(\sigma_{t}\right)=e_{h}\left(\sigma_{t}\right)+\operatorname{div}_{\sigma_{t}} \bar{\theta}_{h}(0)
$$

By assumption, asset dividends are stationary, and efficiency guarantees that equilibrium consumptions are stationary, so (2) entails that $h$ 's endowments must be stationary as well.

\section{A Numerical Example}

A simple example may make the point more clearly. The underlying Markov process is a fair coin toss; that is, $\mathbb{S}=\{1,2\}$, the initial state is 1 , and all transition probabilities are equal to .5. There are two assets, with dividend processes

$$
d^{j}(s)=\left\{\begin{array}{l}
1 \text { if } j=s \\
0 \text { if } j \neq s
\end{array}\right.
$$


There are two agents, with log utility and a common discount factor $\beta=2 / 3$. Individual endowments depend on the underlying state and on whether the date is even or odd:

$$
\begin{aligned}
& e_{1}(s, t)= \begin{cases}4 & \text { if } s=1 ; t \text { even } \\
6 & \text { if } s=2 ; t \text { even } \\
2 & \text { if } s=1 ; t \text { odd } \\
9 & \text { if } s=2 ; t \text { odd }\end{cases} \\
& e_{2}(s, t)=\left\{\begin{array}{lll}
3 & \text { if } s=1 ; t \text { even } \\
9 & \text { if } s=2 ; t \text { even } \\
5 & \text { if } s=1 ; t \text { odd } \\
6 & \text { if } s=2 ; t \text { odd }
\end{array}\right.
\end{aligned}
$$

We abuse notation and write $e_{h}(s, E), e_{h}(s, O)$ for $h$ 's endowment when the underlying state is $s$ and the date is even/odd respectively. Initial portfolio holdings are

$$
\theta_{1}\left(0^{-}\right)=(0.4,1), \theta_{2}\left(0^{-}\right)=(0.6,0)
$$

Note that the aggregate endowment $\bar{e}=\sum\left[e_{h}+\operatorname{div} \theta_{h}\right]$ is stationary:

$$
\bar{e}(s)=\bar{e}(s, t)=\left\{\begin{array}{rll}
8 & \text { if } & s=1 \\
16 & \text { if } & s=2
\end{array}\right.
$$

There is a (unique) efficient equilibrium, which necessarily has the property that portfolio choices depend only on the underlying state and on time. To find the equilibrium, begin with the implications of the budget equations (1) at the various state/time pairs. To simplify notation, write prices and consumptions as functions of the underlying state and write endowments and portfolio choices as functions of the underlying state and the parity (even or odd) of the time index. For each history $\sigma$, there are budget constraints at each of the two immediately succeeding histories, Because there are four state/time-parity possibilities for $\sigma-(1, E),(2, E),(1, O),(2, O)$ - we obtain 4 pairs of equations. To ease the notational burden, we temporarily suppress the agent subscript: 


$$
\begin{aligned}
& x(1)=e(1, O)+\operatorname{div}_{1} \theta(1, E)+q(1) \cdot \theta(1, E)-q(1) \cdot \theta(1, O) \\
& x(2)=e(2, O)+\operatorname{div}_{2} \theta(1, E)+q(2) \cdot \theta(1, E)-q(2) \cdot \theta(2, O) \\
& x(1)=e(1, O)+\operatorname{div}_{1} \theta(2, E)+q(1) \cdot \theta(2, E)-q(1) \cdot \theta(1, O) \\
& x(2)=e(2, O)+\operatorname{div}_{2} \theta(2, E)+q(2) \cdot \theta(2, E)-q(2) \cdot \theta(2, O) \\
& x(1)=e(1, E)+\operatorname{div}_{1} \theta(1, O)+q(1) \cdot \theta(1, O)-q(1) \cdot \theta(1, E) \\
& x(2)=e(2, E)+\operatorname{div}_{2} \theta(1, O)+q(2) \cdot \theta(1, O)-q(2) \cdot \theta(2, E) \\
& x(1)=e(1, E)+\operatorname{div}_{1} \theta(2, O)+q(1) \cdot \theta(2, O)-q(1) \cdot \theta(1, E) \\
& x(2)=e(2, E)+\operatorname{div}_{2} \theta(2, O)+q(2) \cdot \theta(2, O)-q(2) \cdot \theta(2, E)
\end{aligned}
$$

There is also an initial condition, the date 0 budget constraint:

$$
x(1)=e(1, E)+\operatorname{div}_{1} \theta\left(0^{-}\right)+q(1) \cdot \theta\left(0^{-}\right)-q(1) \cdot \theta(1, E)
$$

Observe immediately that equations (3) and (5), (4) and (6), (7) and (9), (8) and (10), taken together in pairs, imply that portfolio dividends, and hence portfolio choices, depend on time (parity) but not on the underlying state: $\theta(1, E)=\theta(2, E), \theta(1, O)=\theta(2, O)$.

Efficiency entails that each agent consumes a constant fraction of the aggregate endowment:

$$
\begin{aligned}
& x_{1}(s)=\lambda \bar{e}(s) \\
& x_{2}(s)=(1-\lambda) \bar{e}(s)
\end{aligned}
$$

Intertemporal consumption prices can be computed directly from marginal rates of substitution, and asset prices can be computed directly from the familiar stochastic Euler equations:

$$
\begin{aligned}
q_{1}(1) & =\frac{1}{2} \beta\left[1+q_{1}(1)\right]+\frac{1}{4} \beta q_{1}(2) \\
q_{1}(2) & =\beta\left[1+q_{1}(1)\right]+\frac{1}{2} \beta q_{1}(2) \\
q_{2}(1) & =\frac{1}{2} \beta q_{2}(1)+\frac{1}{4} \beta\left[1+q_{2}(2)\right] \\
q_{2}(2) & =\beta q_{2}(1)+\frac{1}{2} \beta\left[1+q_{2}(2)\right]
\end{aligned}
$$


To solve for equilibrium, first recall that $\beta=2 / 3$ and solve the stochastic Euler equations (14) - (17) to conclude that equilibrium asset prices are:

$$
\bar{q}(1)=\left(1, \frac{1}{2}\right) ; \bar{q}(2)=(2,1)
$$

Then use equations (3) - (10) to eliminate all the unknowns except $\lambda$, and finally use the initial condition (11) to solve for $\lambda$. Straightforward algebra gives $\lambda=1 / 2$, so equilibrium consumptions are

$$
\bar{x}_{1}(1)=\bar{x}_{2}(1)=4 ; \bar{x}_{1}(2)=\bar{x}_{2}(2)=8
$$

and the equilibrium portfolio choices are

$$
\begin{aligned}
& \bar{\theta}_{1}(1, E)=\bar{\theta}_{1}(2, E)=(+1.9,-1.2) ; \bar{\theta}_{1}(1, O)=\bar{\theta}_{1}(2, O)=(+0.1,+2.2) \\
& \bar{\theta}_{2}(1, E)=\bar{\theta}_{2}(2, E)=(-0.9,+2.2) ; \bar{\theta}_{2}(1, O)=\bar{\theta}_{2}(2, O)=(+0.9,-1.2)
\end{aligned}
$$

Notice that assets are traded at every history. For instance, consider a history $\sigma$ at which the underlying state is 1 and the date is odd. Agent 1 enters the history holding the portfolio $(+1.9,-1.2)$ (i.e., long 1.9 shares of asset 1 and short 1.2 shares of asset 2) and has an endowment of 2 units of consumption. His portfolio yields a dividend of 1.9 units of consumption; he finances an additional 0.1 units of current consumption, bringing the total to 4 units, by his net portfolio trade of $(-1.8,3.4)$. Agent 2 enters the history holding the portfolio $(-0.9,+2.2)$ and has an endowment of 5 units of consumption. From her endowment she pays the dividend debt of 0.9 units of consumption on her portfolio, consumes 4 units in the current history, and uses the remaining 0.1 units of consumption to finance her net portfolio trade of $(1.8,-3.4)$. Notice that (dollar) volume of trade is 1.8 for asset 1 and 1.7 for asset 2, and that the turnover ratios are large: 1.8 and 3.4, respectively.

\section{History-dependence}

In the example above, individual endowments depend on the underlying state and on time but not on the whole history, and as a consequence, portfolios depend only on time. (We leave it to the reader to verify that this is a general property and not an accident of the example.) However, if individual endowments depend on the whole history then portfolios will depend on the 
whole history as well. To see this, fix histories $\sigma, \tau$ of length $t$ such that the underlying state is the same at $\sigma, \tau$ (say state 1 ) and the underlying state is the same at $\sigma^{-}, \tau^{-}$. By the usual arguments, prices $p$ and consumptions $x$ depend only on the underlying state. Applying the budget equation (1) in the histories $\sigma, \tau$ gives:

$$
\begin{aligned}
& x(1)+q(1) \cdot \theta(\sigma)=e(\sigma)+\operatorname{div}_{1} \theta\left(\sigma^{-}\right)+q(1) \cdot \theta\left(\sigma^{-}\right) \\
& x(1)+q(1) \cdot \theta(\tau)=e(\tau)+\operatorname{div}_{1} \theta\left(\tau^{-}\right)+q(1) \cdot \theta\left(\tau^{-}\right)
\end{aligned}
$$

Subtracting and collecting terms gives

$$
\begin{aligned}
e(\sigma)-e(\tau)=q(1) & \cdot[\theta(\sigma)-\theta(\tau)] \\
& -q(1) \cdot\left[\theta\left(\sigma^{-}\right)-\theta\left(\tau^{-}\right)\right] \\
& -\operatorname{div}_{1}\left[\theta\left(\sigma^{-}\right)-\theta\left(\tau^{-}\right)\right]
\end{aligned}
$$

If $e(\sigma) \neq e(\tau)$ then the left-hand side is not 0 so the right-hand side cannot be 0 either. Hence it cannot be that both $\theta(\sigma)=\theta(\tau)$ and $\theta\left(\sigma^{-}\right)=\theta\left(\tau^{-}\right)$. In either case, we conclude that $\theta$ depends on the history and not just on the date and underlying state.

\section{Conclusion}

Working in a heterogeneous version of the Lucas asset-pricing model, JKS show that if asset dividends and individual endowments are stationary then there is no trade after the initial date. This paper shows that if asset dividends and aggregate endowments are stationary but individual endowments are not stationary then there will be trade after the initial date, and there may be trade at every history.

JKS also compare their no-trade result with the conclusions of continuoustime models such as Merton (1971) that imply a great deal of trade. However, the comparison does not seem an apt one to us. In the continuous-time model, trade takes place because information about the future of the dividend is revealed gradually (this is equally true in general equilibrium settings such as Duffie \& Huang (1985) and Duffie \& Zame (1989) as in more common partial equilibrium settings). In the Lucas framework, stationarity means that all information about the future of the dividend process is known at the initial date. 


\section{References}

J. Darrell Duffie, Security Markets: Stochastic Models, London: Academic Press (1988).

J. Darrell Duffie and Chi-Fu Huang, "Implementing Arrow-Debreu Equilibria by Continuous Trading of Few Long-Lived Securities," Econometrica 53 (1985), 1337-1356.

J. Darrell Duffie and William R. Zame, "The Consumption-based Capital Asset Pricing Model," Econometrica 57 (1989), 1279-1298.

Kenneth Judd, Felix Kubler and Karl Schmedders, "Asset Trading Volume in Infinite-Horizon Economies with Dynamically Complete Markets and Heterogeneous Agents," Journal of Finance 58 (2003), 2203-2217.

Robert Lucas, "Asset Prices in an Exchange Economy," Econometrica 46 (1978), 1429-1445.

Robert Merton, "Optimum Consumption and Portfolio Rules in a Continuous-Time Model," Journal of Economic Theory 3 (1971), 9297. 